\title{
Update on Bronchiolitis Obliterans Syndrome in Lung Transplantation
}

\author{
Christine M. Lin • Martin R. Zamora
}

Published online: 12 September 2014

(C) Springer International Publishing AG 2014

\begin{abstract}
Lung transplantation has become an important therapeutic option for patients with end-stage organ dysfunction; however, its clinical usefulness has been limited by the relatively early onset of chronic allograft dysfunction and progressive clinical decline. Obliterative bronchiolitis is characterized histologically by luminal fibrosis of the respiratory bronchioles and clinically by bronchiolitis obliterans syndrome (BOS) which is defined by a measured decline in lung function based on forced expiratory volume $\left(\mathrm{FEV}_{1}\right)$. Since its earliest description, a number of risk factors have been associated with the development of BOS, including acute rejection, lymphocytic bronchiolitis, primary graft dysfunction, infection, donor specific antibodies, and gastroesophageal reflux disease. However, despite this broadened understanding, the pathogenesis underlying BOS remains poorly understood and once begun, there are relatively few treatment options to battle the progressive deterioration in lung function.
\end{abstract}

Keywords Lung transplantation · Chronic lung allograft dysfunction $\cdot$ Bronchiolitis obliterans syndrome

\section{Introduction}

Lung transplantation has become an important therapeutic option for patients with end-stage organ dysfunction. The

\section{M. Lin}

University of Colorado, Denver - Anschutz Medical Campus, 12700 East 19th Avenue, Room 9470E, Aurora, CO 80045, USA e-mail: christine.lin@ucdenver.edu

\section{R. Zamora ( $\bowtie)$}

University of Colorado, Denver - Anschutz Medical Campus,

1635 Aurora Court, Room 7082, Mail Stop F749, Aurora,

CO 80045 , USA

e-mail: marty.zamora@ucdenver.edu one-year adjusted graft survival rate has steadily increased within the last ten years and is now greater than $80 \%$; but despite this "short-term" success, long-term outcomes have failed to attain the longevity similar to that of other solid organ transplants $[1,2]$. Recipients of pulmonary allografts fare far worse than those of other solid organs; per current International Society for Heart and Lung Transplantation (ISHLT) registry data, approximately $50 \%$ of patients develop evidence of chronic dysfunction within five years of transplantation and have a notable decline in survival after that time [3•]. In fact, chronic rejection accounts for greater than $40 \%$ of the "late deaths" that occur in the first year after transplantation [4].

When first elucidated, chronic lung allograft dysfunction (CLAD) was dominated by physiologic descriptions of obstructive lung disease and histopathologically by obliterative bronchiolitis (OB). In fact, CLAD was considered synonymous with bronchiolitis obliterans syndrome (BOS). Howev$\mathrm{er}$, it is being increasingly recognized that CLAD is actually a universal label that should be applied to patients whose forced expiratory volume $\left(\mathrm{FEV}_{1}\right)$ and/or forced vital capacity (FVC) are less than or equal to $80 \%$ of baseline for greater than or equal to three weeks, but that for further clarification, patients should then be subdivided based on physiology (Fig. 1). Specifically, patients can fall within a "restrictive CLAD" phenotype (restrictive allograft syndrome) or an "obstructive CLAD" phenotype (BOS) with acknowledgement that there may be some overlap between the two forms [5]. This review will focus on "obstructive CLAD" or BOS with discussion of its diagnosis, risk factors, pathogenesis, and treatment.

\section{Diagnosis of BOS}

In early cohorts of lung and heart-lung transplant patients, a diagnosis of chronic rejection, or obliterative bronchiolitis, 
Fig. 1 Evaluation of a lung transplant recipient's decline in FEV1. This may be secondary to an identifiable cause (e.g., acute rejection, infection, anastomotic stricture) and lung function may completely normalize with treatment of the underlying pathology. However, if the patient's lung function decline remains for at least three weeks, it is suggestive of chronic lung allograft dysfunction (CLAD). If the FEV 1 declines to $\leq 80 \%$ of the recipient's best post-

transplantation values despite treatment or without identifying a clear cause, then a specific CLAD phenotype (restrictive or obstructive) should be delineated based on the recipient's pulmonary function tests. These forms of lung dysfunction, however, are not mutually exclusive and a patient may have features of both phenotypes. Abbreviations: FEVI - forced expiratory volume in one second, FVC-forced vital capacity, TLC - total lung capacity

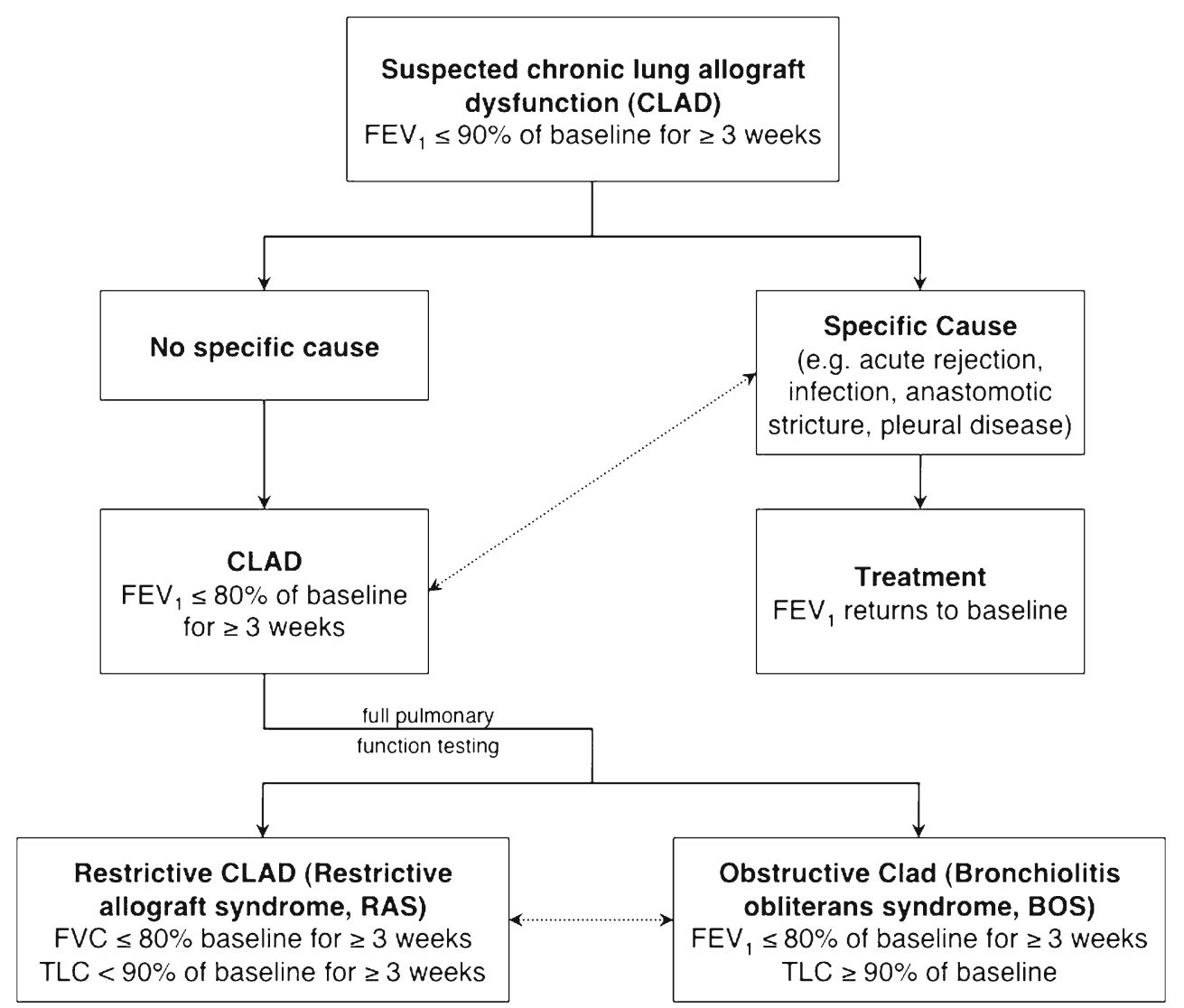

was made via tissue specimens. From a histopathological standpoint, $\mathrm{OB}$ is characterized by the presence of luminal fibrosis in the respiratory bronchioles [6]. It is suspected that this fibrotic process is first initiated by a lymphocytic infiltrate into the submucosa of the airway due to an inflammatory insult; this then leads to localized necrosis with denudation of the respiratory epithelium [7]. Subsequently, the increase of non-specific inflammatory mediators leads to chemotaxis of other cells, including neutrophils, which then stimulate the migration of fibroblasts and myofibroblasts into the airway lumen. Eventually, the formation of intraluminal granulation tissue and fibrous plaques results, leading to obliteration of the airways due to vascular and scar tissue formation [8]. Of note, this process is often temporally and regionally heterogeneic within the allograft.

Historically, due to the requirement for histopathology, the diagnosis of chronic rejection was often difficult to make. This was due to the fact that histologic confirmation was challenging to obtain because transbronchial biopsy specimens lacked the sensitivity required for diagnosis and surgical lung biopsy often carried with it a high morbidity and mortality [9]. Therefore, in 1993, a committee sponsored by the ISHLT proposed a clinical description of $\mathrm{OB}$, termed bronchiolitis obliterans syndrome (BOS), which was based on a patient's post-transplant $\mathrm{FEV}_{1}$ [10]. In the 2002 update, the clinical diagnostic criteria for BOS were revised and a "potential" stage of BOS 0 -p was added, which was defined by a $10 \%$ to $19 \%$ decrease in $\mathrm{FEV}_{1}$ and/or by a $\geq 25 \%$ decrease in forced expiratory flow ( $\left.\mathrm{FEF}_{25-75 \%}\right)$ from baseline. This potential BOS stage was designed to alert physicians as to the need for closer functional monitoring of patients who would be a higher risk for clinical decline [11]. BOS was now able to be defined through standardized and easily obtainable and reproducible spirometric methods. Numerous studies have looked at other surrogate markers of BOS, including bronchoalveolar lavage (BAL) neutrophilia, BAL biomarker levels (IL-8, MMP), exhaled nitric oxide, radiologic findings (air-trapping on high-resolution CT chest), bronchial hyperresponsiveness via methacholine challenge, and circulating fibrocyte level; but none of these are routinely used in clinical practice for diagnosis [11-16].

\section{Risk Factors for BOS}

Acute Rejection and Lymphocytic Bronchiolitis

Acute rejection (AR) has repeatedly been shown to be a leading risk factor for BOS in multiple studies and is arguably the most prominent risk factor that has been identified in the literature [17-23]. AR can vary in its clinical presentation; however, if it is also associated with a spirometrically 
significant acute decrease in FEV1 $\geq 10 \%$ of baseline function, these patients are at an even greater risk for BOS and death [24]. Several studies have shown that large airway inflammation, or lymphocytic bronchiolitis, is also significantly associated with BOS [6] and it has been shown to be a risk factor that is independent of acute rejection [25]. Due to this association, it has also been suggested that the presence of lymphocytic bronchiolitis on endobronchial biopsies (even if obtained in clinically asymptomatic patients) may indicate an increased risk for BOS and this population should be targeted for aggressive management to prevent allograft decline [26].

\section{Ischemic and Vascular Injury}

All solid organs undergo a period of ischemia prior to transplantation; data from small studies have generally shown that neither warm nor cold ischemia time has been significantly correlated with BOS [27]. However, vascular-mediated "injury" to the newly transplanted allograft can also occur in the form of primary graft dysfunction (PGD) or ischemia/ reperfusion injury. This form of acute lung injury occurs in $10-25 \%$ of patients after transplantation and is graded clinically based on the $\mathrm{PaO}_{2} / \mathrm{FIO}_{2}$ ratio and appearance of radiographic infiltrates on chest imaging [28]. Early studies regarding the association between PGD and BOS were conflicting, but recent work has concluded that there is a direct relationship between the severity of PGD and the risk of BOS that was independent of other recognized risk factors, including acute rejection [29]. Lastly, ischemia to the airway can also take a "chronic" form due to the nature of the surgical operation. Normally, the lung has a dual blood supply composed of the pulmonary and bronchial arterial circulations, but routinely, only the pulmonary circulation undergoes re-anastomosis. In a landmark European study, coupled with data from one North American center, Pettersson et al., described superior 5-year and 10-year survival in patients who underwent bronchial artery revascularization (BAR) at the time of transplantation. For this group, success improved airway healing as compared to controls and BOS was delayed for two years or greater [30, 31]. This has led to the suggestion, but not widely adopted practice, of performing BAR at the time of transplantation to reduce the incidence of airway ischemic complications in the future.

\section{Infection}

Of the multitude of pulmonary infections that affect lung transplant patients, cytomegalovirus (CMV) has been the most widely studied. However, despite being a suspected contributor to chronic rejection, early studies assessing the impact of CMV infection on BOS were inconclusive due to the wide range of clinical definitions that were utilized [6]. Since that time, however, multiple studies have shown that
CMV is significantly associated with BOS development, and CMV prophylaxis has been clearly associated with a decreased the rate of BOS [32-34]. This may be secondary to a lower incidence of clinically apparent disease, such as CMV pneumonitis. However, with the advent of molecular diagnostic tools (polymerase chain reaction, or PCR) for detection of subclinical CMV replication, even asymptomatic viral activity was significantly associated with the development of BOS [35]. Subsequently, this has raised the question as to the exact timing and length of prophylaxis, especially in high-risk individuals.

Interestingly, relatively few studies have looked at the association between other pulmonary infections and BOS. Timing of infections is important, as pneumonias caused by Gram-positive, Gram-negative, and fungal pathogens are independent determinants of BOS; especially if they occur within the first 100 days of transplantation [36]. One study also reported an association between worsening BOS stage if infection occurred after the start of allograft decline [37]. Even though lung transplant patients are particularly susceptible to complications from respiratory viral infections, analysis of preexisting studies have failed to confirm a true association between respiratory viral infections (influenza, respiratory syncytial virus, parainfluenza, human metapneumovirus, rhinovirus, coronavirus, and adenovirus) with either acute rejection or the development of BOS [38].

Airway colonization, and not necessarily infection, by pathogenic organisms has also been closely linked with BOS development. Vos et al., showed in a retrospective study that postoperative colonization of the airway with Pseudomonas aeruginosa was an independent risk factor for BOS Stage $\geq 1$ and is associated with worse BOS-free survival; especially in patients who had cystic fibrosis as the underlying cause of their end-stage of lung disease [39]. De novo colonization by Pseudomonas aeruginosa was also strongly associated with the subsequent development of BOS [40]. Of fungal infections, colonization by Aspergillus species has been shown to be a risk factor of BOS independent of acute rejection [41]. This finding was later verified in a two-center study that specified small conidia species (Aspergillus fumigatus, A. nidulans, A. terreus, and A. flavipes) were significantly associated with BOS. Colonization with these small conidia species was also associated with an increased risk of death [41]. Hsu et al., hypothesized that Aspergillus, with its capacity to grow in hypoxic environments, could modulate the host immune and repair response in an allograft; they demonstrated in a murine tracheal transplant model that $A$. fumigatus invasion increased with progressive airway ischemia and in turn, worsened regional perfusion to infected areas [42]. However, investigation of the lung microbiome from a molecular sequencing standpoint argues that the development of BOS is not necessarily linked to specific organisms, but rather the acquisition of novel and atypical microbial populations that 
are altered from the host's native pre-transplant microbiota [43].

Antibodies and Antibody Mediated Rejection

Within recent years, the clinical significance of donor-specific antibodies (DSA) in the pathogenesis of chronic allograft dysfunction has received increasing attention. Early on, the association between BOS and human leukocyte antigen (HLA) mismatches at the A, B, and DR loci was not clear, as the majority of the evidence was conflicting in nature and limited by single-center studies with inadequate power. HLA Class I and II mismatch between donor and recipient, development of anti-HLA Class I antibodies after lung transplantation, and an increased frequency of allo-reactive CD4+ T cells had been identified as significant risk factors for the development of BOS in these studies [44-47]; however, a large retrospective analysis of lung registry data failed to show a consistent association [48]. Thus, the presence of DSA against HLA antigens as defined by a positive panel reactive antibody (PRA) screen was felt to be only a "potential" risk factor for BOS [46]. Nonetheless, since that time, Chalermskulrat et al., showed that the number of combined HLA-A and HLA-B mismatches were strongly associated with BOS stage with a trend towards significance seen with HLA-DR mismatches [49]. Numerous clinical studies have also shown that DSA is undoubtedly associated with acute antibody mediated rejection (AMR) and BOS [50-54]. Antibodies to non-HLA antigens have been implicated pathogenesis of BOS as well. Hagedorn et al., showed that a "profile" of autoantibodies was able to distinguish patients with BOS Stage 0/1 from BOS Stage 2/3 [55]. In fact, "auto-allo immunity" to cryptic self-antigens such as Type V collagen and $\mathrm{K}-\alpha 1$ tublin are present in a significant number of patients with BOS [56-62]; although it is unclear if these antibodies are acting to directly injury the epithelium, or merely serve as a marker for ongoing immunologic damage.

\section{Gastroesophageal Reflux Disease}

Gastroesophageal reflux disease (GERD) was first identified as a hypothetical risk factor [11,63] due to the postulation that aspiration of acid could cause direct injury to the respiratory epithelium. Early case reports suggested that aggressive treatment of reflux could lead to a reversal in a patient's clinical decline [64]. In a key study, Davis et al. showed that performing a Nissen fundoplication in patients with documented GERD after lung transplantation improved survival and allograft function and decreased BOS stage [65]. However, Blondeau et al. showed that patients with GERD did not necessarily have an increased risk for BOS, but those with elevated levels of bile acid in BAL fluid did, suggesting that it was not necessarily the presence of GERD that would predispose a patient to BOS, but rather the concurrent act of aspiration that would lead to an increased risk for disease [66]. Since that study, GERD, as assessed via $\mathrm{pH}$ monitoring, impedance manometry, and esophagogastroduodenoscopy (EGD) has been shown to be associated with the development of BOS and BOS-free survival [67]. This group also showed that patients with significant reflux also had an increased delayed-type hypersensitivity reaction to Type $\mathrm{V}$ collagen, suggesting that acid not only causes direct injury to the respiratory epithelium, but can also unmask cryptic antigens that can further drive a damaging immunologic response. However, although widely employed, the use of a proton-pump inhibitor (PPI) has not been shown to prevent non-acid reflux and gastric aspiration [66].

\section{Donor-Related Factors}

Numerous retrospective studies have looked at donorrelated factors and whether or not they are associated with the development of BOS. In one of the largest retrospective reviews of the UNOS database $(6,991$ patients), the authors showed that donor-related factors significantly associated with BOS at five-years posttransplantation included receiving an organ from an older donor ( $>60$ years), a recent smoker (smoked/quit within six months of donation), or from an individual with a history of myocardial infarction. Interestingly, a high donor $\mathrm{PaO} 2$ was also associated with a higher rate in the development of BOS [68]. Donor gender has also been studied; trends for increased survival were demonstrated in gender mismatched recipients, with male-tomale transplant recipients having the worst overall survival $[17,69]$. Female-to-female recipient donor combinations were independently associated with increased freedom from BOS and longer duration of BOS-free survival relative to the male-to-male recipient donor combination, but the adjusted cumulative incidence of BOS was similar [17]. Nonetheless, this finding has not specifically changed organ allocation to particular recipients.

\section{Other Factors}

Lastly, in contrast to the majority of other solid organ transplants, the lung is in direct contact with the environment. Nawrot et al., described that patients who lived within 171 meters (the lowest tertile) of a major road (defined as a highway, national road, or large local road) were approximately twice as likely to develop BOS and twice as likely to die than patients living further away [70], again pointing to the association between environmental exposure and direct injury with a decline in allograft dysfunction. 


\section{Pathogenesis of BOS}

Despite elucidation of the numerous risk factors outlined above, the exact pathogenesis of BOS has not been elucidated. It is likely, however, that injury, remodeling, and repair does not occur in a step-wise fashion, but is rather a complex milieu of disordered interactions. For instance, patients with PGD have higher levels of inflammatory markers (MCP-1, IL-10, IL-1 $\beta$, IL-2, IFN- $\gamma$, and IL-12) in the early post-transplant period in comparison to patients without PGD. Interestingly, these patients also developed anti-HLA antibodies at a higher rate, which would then put them at higher risk for both acute cellular rejection, antibody-mediated rejection, and lymphocytic bronchiolitis [71-73]. Thus, the "bottom line" is that the pathophysiological association between these factors can be linked through a common pathway of upregulation of inflammatory cytokines and cellular trafficking that leads to further epithelial and vascular damage. Notably, BOS has been closely linked with the dysfunction of Clara cells, which are secretory cells present in the bronchial epithelium that contribute to host defense and serve as the progenitor cell to repair the respiratory epithelium after injury [74-76]. Repeated injury, through any mechanism, will contribute to continued inflammation, changes in $\mathrm{T}$ cell phenotypes, unmasking of selfantigens, and neoangiogeneis that then likely culminates in a robust, but deranged, repair process and chronic remodeling of the airway.

\section{Prevention \& Treatment of BOS}

As described, the development of BOS can be influenced by a multitude of factors and is the result of both direct and indirect injury to the respiratory epithelium and vasculature. Therefore, it is not surprising there are relatively few treatment modalities that have been shown to effectively slow or reverse this process. Modulation of the immune response from the time of transplantation has been investigated; studies in induction therapy in lung transplantation have failed to show a consistent benefit in terms of reducing long-term complications and the incidence of BOS [17, 77-83]. In the posttransplant period, BOS has been treated with a variety of medical regimens that have included, but are not limited to, augmentation of immunosuppression with corticosteroids, methotrexate, cyclophosphamide, sirolimus, and antilymphocyte antibody preparations. No specific maintenance immunosuppressive regimen or protocol has been shown to be superior at reducing the incidence of BOS [4], however, metaanalyses suggest a trend towards lower risk in patients receiving tacrolimus (versus cyclosporine) in a calcineurin-inhibitor based regimen [84, 85]. This result was demonstrated in a multicenter, international prospective trial, which reported that use of a de novo tacrolimus-based regimen significantly reduced the risk for BOS Stage $\geq 1\left[86^{\bullet}\right]$. Nonetheless, all of these results were limited by small sample size and relative lack of randomized controls, thus, there are currently no formalized recommendations regarding the use of one calcineurin inhibitor over another.

In general, the airway changes in BOS are considered to be irreversible once they occur. However, some cases may respond to specific treatment. For instance, if a large part of a patient's decline is due to a specific etiology (e.g., GERD), then specific measures aimed at treating this underlying cause may ameliorate or reverse the decline in lung function. However, as a general cohort of patients, several single-center studies have shown that a significant proportion of patients with BOS respond to azithromycin with an increase in FEV1 of at least $10 \%$; some patients even showed a complete reversal of their prior physiologic decline with return to BOS Stage 0 [87-89]. The role of macrolides in the prevention of BOS has also been examined; Vos et al., studied a cohort of patients in which therapy with azithromycin versus placebo was initiated at the time of discharge from their initial hospitalization for transplantation; patients were then maintained on the medication for two years. In this study, the development of BOS occurred significantly less in patients receiving chronic azithromycin therapy $(12.5 \%$ versus $44.2 \%$ ) [90]. In contrast, however, Dhillon et al., showed that routine use of clarithromycin was actually associated with an increased risk in developing BOS with no significant change in 5-year survival [91].

Rescue therapies have also been employed and include extracorporeal photopheresis (ECP), a treatment in which white blood cells isolated from the recipient are exposed to photoactivatable 8-methoxypsoralen and ultraviolet A radiation. The cells are subsequently returned to the patient and appear to induce a cytotoxic effect on T-cell formation [92]. It was first reported in a "Letter to the Editor" which described its use in three patients with good clinical response [93], and since that time, several other single-center studies have consistently demonstrated that ECP therapy is relatively welltolerated and slows the rate of FEV1 decline [94-96]. However, this therapy has yet to be utilized in widespread clinical practice. Re-transplantation remains as the only definitive treatment for advanced BOS, even with the acknowledgement that outcomes tend to be poor [3•].

\section{Conclusion}

It has been over 50 years since the first human lung transplant was performed in 1963, yet chronic lung allograft dysfunction due to bronchiolitis obliterans syndrome still represents the major hurdle to long-term allograft and patient survival. As outlined above, there are numerous risk factors that can predispose patients to the development of this process; however, 
its pathogenesis remains poorly understood. This is reflected in heterogeneity of presentation in which some patients may experience a rapid decline, while others experience a slow, but progressive, deterioration in lung function. It is suspected that inflammatory conditions, whether arising from infection, ischemic injury, or exposure can cause direct and indirect injury, leading to a disordered repair of the airway epithelium. Very few treatment options exist once BOS becomes clinically evident, and no specific induction or maintenance immunosuppressive regimen has proven superior to prevent its onset. As such, re-transplantation remains the only definitive treatment for this disease. Therefore, it is hoped that further understanding of the mechanisms underlying this process will allow lung transplantation to be a viable long-term therapeutic option for patients with end-stage lung disease.

\section{Compliance with Ethics Guidelines}

Conflict of Interest Christine M. Lin and Martin R. Zamora declare no conflicts of interest.

Human and Animal Rights and Informed Consent All studies by the authors involving animal and/or human subjects were performed after approval by the appropriate institutional review boards. When required, written informed consent was obtained from all participants.

\section{References}

Papers of particular interest, published recently, have been highlighted as:

- Of importance

1. Kwun J, Knechtle SJ. Overcoming chronic rejection-can it B? Transplantation. 2009;88(8):955-61.

2. Organ Procurement and Transplantation Network (OPTN) and Scientific Registry of Transplant Recipients (SRTR). OPTN/ SRTR 2010 Annual Data Report, Department of Health and Human Services, Health Resources and Services Administration, Healthcare Systems Bureau, Division of Transplantation: Rockville; 2010

3. Yusen RD et al. The Registry of the International Society for Heart and Lung Transplantation: thirtieth adult lung and heart-lung transplant report-2013; focus theme: age. J Heart Lung Transplant. 2013;32(10):965-78. This document, published by the International Society for Heart and Lung Transplantation and updated yearly, describes donor and recipient characteristics, transplant type, and recipient outcomes data for lung tranplants occurring from 1988 to the present.

4. Hachem RR, Trulock EP. Bronchiolitis obliterans syndrome: pathogenesis and management. Semin Thorac Cardiovasc Surg. 2004;16(4):350-5.

5. Verleden GM et al. A new classification system for chronic lung allograft dysfunction. J Heart Lung Transplant. 2014;33(2):127-33.

6. Sharples LD et al. Risk factors for bronchiolitis obliterans: a systematic review of recent publications. J Heart Lung Transplant. 2002;21(2):271-81.
7. Yousem SA. Lymphocytic bronchitis/bronchiolitis in lung allograft recipients. Am J Surg Pathol. 1993;17(5):491-6.

8. Yousem SA, Duncan SR, Griffith BP. Interstitial and airspace granulation tissue reactions in lung transplant recipients. Am J Surg Pathol. 1992;16(9):877-84.

9. Kramer MR et al. The diagnosis of obliterative bronchiolitis after heart-lung and lung transplantation: low yield of transbronchial lung biopsy. J Heart Lung Transplant. 1993;12(4):675-81.

10. Cooper JD et al. A working formulation for the standardization of nomenclature and for clinical staging of chronic dysfunction in lung allografts. International Society for Heart and Lung Transplantation. J Heart Lung Transplant. 1993;12(5):713-6.

11. Estenne $\mathrm{M}$ et al. Bronchiolitis obliterans syndrome 2001: an update of the diagnostic criteria. J Heart Lung Transplant. 2002;21(3):297310 .

12. Kennedy VE, Todd JL, Palmer SM. Bronchoalveolar lavage as a tool to predict, diagnose and understand bronchiolitis obliterans syndrome. Am J Transplant. 2013;13(3):552-61.

13. Vos $\mathrm{R}$ et al. Bronchoalveolar lavage neutrophilia in acute lung allograft rejection and lymphocytic bronchiolitis. J Heart Lung Transplant. 2010;29(11):1259-69.

14. DiGiovine B et al. Bronchoalveolar lavage neutrophilia is associated with obliterative bronchiolitis after lung transplantation: role of IL-8. J Immunol. 1996;157(9):4194-202.

15. Riise GC et al. Persistent high BAL fluid granulocyte activation marker levels as early indicators of bronchiolitis obliterans after lung transplant. Eur Respir J. 1999;14(5):1123-30.

16. LaPar DJ et al. Circulating fibrocytes correlate with bronchiolitis obliterans syndrome development after lung transplantation: a novel clinical biomarker. Ann Thorac Surg. 2011;92(2):470-7. discussion 477.

17. Burton $\mathrm{CM}$ et al. Acute cellular rejection is a risk factor for bronchiolitis obliterans syndrome independent of post-transplant baseline FEV1. J Heart Lung Transplant. 2009;28(9):888-93.

18. Keller CA et al. Bronchiolitis obliterans in recipients of single, double, and heart-lung transplantation. Chest. 1995;107(4):973-80.

19. Girgis RE et al. Risk factors for the development of obliterative bronchiolitis after lung transplantation. J Heart Lung Transplant. 1996;15(12):1200-8.

20. Bando $\mathrm{K}$ et al. Obliterative bronchiolitis after lung and heart-lung transplantation. An analysis of risk factors and management. J Thorac Cardiovasc Surg. 1995;110(1):4-13. discussion 13-4.

21. Kesten $\mathrm{S}$ et al. Treatment of presumed and proven acute rejection following six months of lung transplant survival. Am J Respir Crit Care Med. 1995;152(4 Pt 1):1321-4.

22. Sharples LD et al. Development of bronchiolitis obliterans syndrome in recipients of heart-lung transplantation-early risk factors. Transplantation. 1996;61(4):560-6.

23. Husain AN et al. Analysis of risk factors for the development of bronchiolitis obliterans syndrome. Am J Respir Crit Care Med. 1999;159(3):829-33.

24. Davis WA et al. Spirometrically significant acute rejection increases the risk for BOS and death after lung transplantation. Am J Transplant. 2012;12(3):745-52.

25. Glanville AR et al. Severity of lymphocytic bronchiolitis predicts long-term outcome after lung transplantation. Am J Respir Crit Care Med. 2008;177(9):1033-40.

26. Greenland JR et al. Association of large-airway lymphocytic bronchitis with bronchiolitis obliterans syndrome. Am J Respir Crit Care Med. 2013;187(4):417-23.

27. Snell GI, Westall GP. The contribution of airway ischemia and vascular remodelling to the pathophysiology of bronchiolitis obliterans syndrome and chronic lung allograft dysfunction. Curr Opin Organ Transplant. 2010;15(5):558-62.

28. Christie JD et al. Report of the ISHLT Working Group on Primary Lung Graft Dysfunction part II: definition. A consensus statement 
of the International Society for Heart and Lung Transplantation. J Heart Lung Transplant. 2005;24(10):1454-9.

29. Huang HJ et al. Late primary graft dysfunction after lung transplantation and bronchiolitis obliterans syndrome. Am J Transplant. 2008;8(11):2454-62.

30. Pettersson GB et al. Comparative study of bronchial artery revascularization in lung transplantation. J Thorac Cardiovasc Surg. 2013;146(4):894-900 e3.

31. Pettersson GB, Yun JJ, Norgaard MA. Bronchial artery revascularization in lung transplantation: techniques, experience, and outcomes. Curr Opin Organ Transplant. 2010;15(5):572-7.

32. Ruttmann E et al. Combined CMV prophylaxis improves outcome and reduces the risk for bronchiolitis obliterans syndrome (BOS) after lung transplantation. Transplantation. 2006;81(10):1415-20.

33. Chmiel $\mathrm{C}$ et al. Ganciclovir/valganciclovir prophylaxis decreases cytomegalovirus-related events and bronchiolitis obliterans syndrome after lung transplantation. Clin Infect Dis. 2008;46(6):8319.

34. Johanssson I, Martensson G, Andersson R. Cytomegalovirus and long-term outcome after lung transplantation in Gothenburg, Sweden. Scand J Infect Dis. 2010;42(2):129-36.

35. Paraskeva $\mathrm{M}$ et al. Cytomegalovirus replication within the lung allograft is associated with bronchiolitis obliterans syndrome. Am J Transplant. 2011;11(10):2190-6.

36. Valentine VG et al. Effect of etiology and timing of respiratory tract infections on development of bronchiolitis obliterans syndrome. $\mathrm{J}$ Heart Lung Transplant. 2009;28(2):163-9.

37. Heng D et al. Bronchiolitis obliterans syndrome: incidence, natural history, prognosis, and risk factors. J Heart Lung Transplant. 1998;17(12):1255-63.

38. Vu DL et al. Respiratory viruses in lung transplant recipients: a critical review and pooled analysis of clinical studies. Am J Transplant. 2011;11(5):1071-8.

39. Vos R et al. Pseudomonal airway colonisation: risk factor for bronchiolitis obliterans syndrome after lung transplantation? Eur Respir J. 2008;31(5):1037-45.

40. Botha $\mathrm{P}$ et al. Pseudomonas aeruginosa colonization of the allograft after lung transplantation and the risk of bronchiolitis obliterans syndrome. Transplantation. 2008;85(5):771-4.

41. Weigt SS et al. Aspergillus colonization of the lung allograft is a risk factor for bronchiolitis obliterans syndrome. Am J Transplant. 2009;9(8):1903-11.

42. Hsu JL et al. Aspergillus fumigatus invasion increases with progressive airway ischemia. PLoS One. 2013;8(10):e77136.

43. Willner DL et al. Reestablishment of recipient-associated microbiota in the lung allograft is linked to reduced risk of bronchiolitis obliterans syndrome. Am J Respir Crit Care Med. 2013;187(6): 640-7.

44. SivaSai KS et al. Indirect recognition of donor HLA class I peptides in lung transplant recipients with bronchiolitis obliterans syndrome. Transplantation. 1999;67(8):1094-8.

45. Jaramillo A et al. Development of ELISA-detected anti-HLA antibodies precedes the development of bronchiolitis obliterans syndrome and correlates with progressive decline in pulmonary function after lung transplantation. Transplantation. 1999;67(8):1155-61.

46. Sundaresan $\mathrm{S}$ et al. HLA-A locus mismatches and development of antibodies to HLA after lung transplantation correlate with the development of bronchiolitis obliterans syndrome. Transplantation. 1998;65(5):648-53.

47. Smith MA et al. Effect of development of antibodies to HLA and cytomegalovirus mismatch on lung transplantation survival and development of bronchiolitis obliterans syndrome. J Thorac Cardiovasc Surg. 1998;116(5):812-20.

48. Quantz MA et al. Does human leukocyte antigen matching influence the outcome of lung transplantation? An analysis of 3,549 lung transplantations. J Heart Lung Transplant. 2000;19(5):473-9.

49. Chalermskulrat $\mathrm{W}$ et al. Human leukocyte antigen mismatches predispose to the severity of bronchiolitis obliterans syndrome after lung transplantation. Chest. 2003;123(6):1825-31.

50. Witt $\mathrm{CA}$ et al. Acute antibody-mediated rejection after lung transplantation. J Heart Lung Transplant. 2013;32(10):1034-40.

51. Maruyama $T$ et al. Induction of obliterative airway disease by anti-HLA class I antibodies. Am J Transplant. 2005;5(9): 2126-34.

52. Hachem R. Antibody-Mediated Lung Transplant Rejection. Curr Respir Care Rep. 2012;1(3):157-61.

53. Lobo LJ et al. Donor-specific antibodies are associated with antibody-mediated rejection, acute cellular rejection, bronchiolitis obliterans syndrome, and cystic fibrosis after lung transplantation. J Heart Lung Transplant. 2013;32(1):70-7.

54. Otten HG et al. Identification of non-HLA target antigens recognized after lung transplantation. J Heart Lung Transplant. 2006;25(12):1425-30.

55. Hagedorn $\mathrm{PH}$ et al. Chronic rejection of a lung transplant is characterized by a profile of specific autoantibodies. Immunology. 2010;130(3):427-35.

56. Hachem RR et al. Antibodies to K-alpha 1 tubulin and collagen V are associated with chronic rejection after lung transplantation. Am J Transplant. 2012;12(8):2164-71.

57. Saini D et al. Alloimmunity-induced autoimmunity as a potential mechanism in the pathogenesis of chronic rejection of human lung allografts. J Heart Lung Transplant. 2011;30(6):624-31.

58. Zhang Q, Reed EF. Non-MHC antigenic targets of the humoral immune response in transplantation. Curr Opin Immunol. 2010;22(5):682-8.

59. Shilling RA, Wilkes DS. Immunobiology of chronic lung allograft dysfunction: new insights from the bench and beyond. Am J Transplant. 2009;9(8):1714-8.

60. Yoshida $\mathrm{S}$ et al. Anti-type V collagen lymphocytes that express IL17 and IL-23 induce rejection pathology in fresh and well-healed lung transplants. Am J Transplant. 2006;6(4):724-35.

61. Sumpter TL, Wilkes DS. Role of autoimmunity in organ allograft rejection: a focus on immunity to type $\mathrm{V}$ collagen in the pathogenesis of lung transplant rejection. Am J Physiol Lung Cell Mol Physiol. 2004;286(6):L1129-39.

62. Goers TA et al. De novo production of K-alpha1 tubulin-specific antibodies: role in chronic lung allograft rejection. J Immunol. 2008;180(7):4487-94.

63. Hadjiliadis D et al. Gastroesophageal reflux disease in lung transplant recipients. Clin Transplant. 2003;17(4):363-8.

64. Palmer SM et al. Gastroesophageal reflux as a reversible cause of allograft dysfunction after lung transplantation. Chest. 2000;118(4): 1214-7.

65. Davis Jr RD et al. Improved lung allograft function after fundoplication in patients with gastroesophageal reflux disease undergoing lung transplantation. J Thorac Cardiovasc Surg. 2003;125(3):533-42.

66. Blondeau $\mathrm{K}$ et al. Gastro-oesophageal reflux and gastric aspiration in lung transplant patients with or without chronic rejection. Eur Respir J. 2008;31(4):707-13.

67. Bobadilla JL et al. Reflux-induced collagen type v sensitization: potential mediator of bronchiolitis obliterans syndrome. Chest. 2010;138(2):363-70.

68. Hennessy SA et al. Donor factors are associated with bronchiolitis obliterans syndrome after lung transplantation. Ann Thorac Surg. 2010;89(5):1555-62.

69. Alvarez A et al. Influence of donor-recipient gender mismatch on graft function and survival following lung transplantation. Interact Cardiovasc Thorac Surg. 2013;16(4):426-35. 
70. Nawrot TS et al. The impact of traffic air pollution on bronchiolitis obliterans syndrome and mortality after lung transplantation. Thorax. 2011;66(9):748-54.

71. Bharat A et al. Immunological link between primary graft dysfunction and chronic lung allograft rejection. Ann Thorac Surg. 2008;86(1):189-95. discussion 196-7.

72. Girnita AL et al. HLA-specific antibodies are risk factors for lymphocytic bronchiolitis and chronic lung allograft dysfunction. Am J Transplant. 2005;5(1):131-8.

73. Girnita AL et al. HLA-specific antibodies are associated with highgrade and persistent-recurrent lung allograft acute rejection. J Heart Lung Transplant. 2004;23(10):1135-41.

74. Stripp BR, Reynolds SD. Maintenance and repair of the bronchiolar epithelium. Proc Am Thorac Soc. 2008;5(3):328-33.

75. Kelly FL et al. Epithelial clara cell injury occurs in bronchiolitis obliterans syndrome after human lung transplantation. Am J Transplant. 2012;12(11):3076-84.

76. Bourdin A et al. Donor clara cell secretory protein polymorphism is a risk factor for bronchiolitis obliterans syndrome after lung transplantation. Transplantation. 2012;94(6):652-8.

77. Ailawadi $\mathrm{G}$ et al. Effects of induction immunosuppression regimen on acute rejection, bronchiolitis obliterans, and survival after lung transplantation. J Thorac Cardiovasc Surg. 2008;135(3):594-602.

78. Brock MV et al. Induction therapy in lung transplantation: a prospective, controlled clinical trial comparing OKT3, anti-thymocyte globulin, and daclizumab. J Heart Lung Transplant. 2001;20(12): 1282-90.

79. Hachem RR et al. The impact of induction on survival after lung transplantation: an analysis of the International Society for Heart and Lung Transplantation Registry. Clin Transplant. 2008;22(5): 603-8.

80. Hartwig MG et al. Rabbit anti-thymocyte globulin induction therapy does not prolong survival after lung transplantation. J Heart Lung Transplant. 2008;27(5):547-53.

81. Mullen JC et al. A randomized, controlled trial of daclizumab vs anti-thymocyte globulin induction for lung transplantation. J Heart Lung Transplant. 2007;26(5):504-10.

82. Palmer SM et al. Rabbit antithymocyte globulin decreases acute rejection after lung transplantation: results of a randomized, prospective study. Chest. 1999;116(1):127-33.

83. Shyu $\mathrm{S}$ et al. Five-year outcomes with alemtuzumab induction after lung transplantation. J Heart Lung Transplant. 2011;30(7):743-54.
84. Fan Y, Xiao YB, Weng YG. Tacrolimus versus cyclosporine for adult lung transplant recipients: a meta-analysis. Transplant Proc. 2009;41(5):1821-4.

85. Penninga $\mathrm{L}$ et al. Tacrolimus versus cyclosporin as primary immunosuppression for lung transplant recipients. Cochrane Database Syst Rev. 2013;5, CD008817.

86. Treede $\mathrm{H}$ et al. Tacrolimus and cyclosporine have differential effects on the risk of development of bronchiolitis obliterans syndrome: results of a prospective, randomized international trial in lung transplantation. J Heart Lung Transplant. 2012;31(8):797-804. This study is a multi-center, prospective, randomized trial to assess for superiority of tacrolimus versus cyclosporine as maintenance immunosuppression in reducing the risk of BOS development.

87. Gerhardt SG et al. Maintenance azithromycin therapy for bronchiolitis obliterans syndrome: results of a pilot study. Am J Respir Crit Care Med. 2003;168(1):121-5.

88. Verleden GM, Dupont LJ. Azithromycin therapy for patients with bronchiolitis obliterans syndrome after lung transplantation. Transplantation. 2004;77(9):1465-7.

89. Yates B et al. Azithromycin reverses airflow obstruction in established bronchiolitis obliterans syndrome. Am J Respir Crit Care Med. 2005;172(6):772-5.

90. Vos $\mathrm{R}$ et al. A randomised controlled trial of azithromycin to prevent chronic rejection after lung transplantation. Eur Respir J. 2011;37(1):164-72.

91. Dhillon GS et al. Clarithromycin for prevention of bronchiolitis obliterans syndrome in lung allograft recipients. Clin Transplant. 2012;26(1):105-10.

92. Knobler R. Extracorporeal photochemotherapy-present and future. Vox Sang. 2000;78 Suppl 2:197-201.

93. Slovis BS, Loyd JE, King Jr LE. Photopheresis for chronic rejection of lung allografts. N Engl J Med. 1995;332(14):962.

94. Benden $\mathrm{C}$ et al. Extracorporeal photopheresis after lung transplantation: a 10-year single-center experience. Transplantation. 2008;86(11):1625-7.

95. Morrell MR et al. The efficacy of photopheresis for bronchiolitis obliterans syndrome after lung transplantation. J Heart Lung Transplant. 2010;29(4):424-31.

96. Jaksch $\mathrm{P}$ et al. A prospective interventional study on the use of extracorporeal photopheresis in patients with bronchiolitis obliterans syndrome after lung transplantation. J Heart Lung Transplant. 2012;31(9):950-7. 